\title{
Embryogenèse somatique chez trois espèces de chênes méditerranéens
}

\author{
C. Féraud-Keller, M. El Maâtaoui, O. Gouin et H. Espagnac \\ Laboratoire de Biologie Végétale Expérimentale, Faculté des Sciences, 33, rue Louis-Pasteur, \\ 84000 Avignon, France
}

\section{Introduction}

Dans le cadre d'une analyse de la multiplication végétative de chênes méditerranéens, nous avons pu obtenir des embryons somatiques chez trois espèces (Quercus suber, El Maâtaoui et Espagnac, 1987; Q. ilex, Féraud-Keller et Espanac, 1989; Q. pubescens). Le type de matériel végétal utilisé et les conditions d'obtention étant différentes pour chaque espèce, il nous a semblé utile d'exposer ces résultats.

\section{Matériel et Méthodes}

Pour le chêne liège ( $Q$. suber) le matériel est fourni par de jeunes individus âgés de 6 à 8 mois obtenus à partir de glands récoltés en Tunisie, cultivés en serre à Avignon et sur lesquels sont prélevées des portions dentrenœeuds d'une longueur d'environ $5 \mathrm{~mm}$. Pour le chêne vert ( $Q$. ilex). les implants sont des frag- ments de limbes pourvus de la nervure principale et des pétioles entiers de feuilles récoltées sur la pousse de l'année d'arbres âgés d'environ 50 ans. Les embryons zygotiques immatures de chêne pubescent ( $Q$. pubescens) et de chêne liège sont prélevés sur des glands provenant de populations naturelles.

Tout ce matériel à l'exception des fragments de chêne vert, est implanté après une décontamination par un trempage rapide dans l'éthanol a $70 \%$ suivi d'un traitement de $25 \mathrm{~min}$ dans l'hypochlorite de calcium à $45 \mathrm{~g} /$ et de trois rinçages à l'eau stérile. Le chène vert lui est stérilisé par un passage de $20 \mathrm{~min}$ dans l'hypochlorite de calcium à $90 \mathrm{~g} / \mathrm{l}$ puis rincé à l'eau stérile.

Plusieurs essais préliminaires nous ont conduits à adopter le milieu de base de Murashige et Skoog contenant, pour le chêne liège et le chêne pubescent, $20 \mathrm{~g} / \mathrm{l}$ de glucose, $8 \mathrm{~g} / \mathrm{l}$ de gélose, $1 \mathrm{~g} / \mathrm{l}$ d'hydrolysat de caséine, $2 \mathrm{mg} / \mathrm{l}$ d'AlB et $2 \mathrm{mg} / \mathrm{l}$ de BAP et, pour le chêne vert. $30 \mathrm{~g} / \mathrm{de}$ saccharose, $8 \mathrm{~g} / \mathrm{l}$ de gélose, $100 \mathrm{mg} / \mathrm{l}$ d'inositol, 0,5 mg!l d'ANA et $4 \mathrm{mg} / \mathrm{l}$ de BAP. Le $\mathrm{pH}$ est ajusté à 56 avant un autoclavage de 15 min à $110^{\circ} \mathrm{C}$. Les fragments sont placés horizontalement à la surface du milieu, en tubes ou en boîtes de Pétri. Les cultures sont placées dans une salle climatisée à $25^{\circ} \mathrm{C}$ sous une photopériode de $16 \mathrm{~h}$.

AlB: acide indolylbutyrique: ANA: acide naphtalène-acétique; BAP: 6-benzylaminopurine; 2,4-D: acide dichlorophénoxyacetique. 
Les contrôles histologiques ont été réalisés sur matériel fixé dans le mélange FAA (formol:acide acétique:éthanol, 10:10:9, V/v), déshydraté dans l'éthanol et inclus dans la paraffine. Les coupes en série, d'une épaisseur de $7 \mu \mathrm{m}$, ont été colorées à l'hématoxylinesafranine-bleu d'aniline.

\section{Résultats}

Chez le chêne liège, 2 semaines après leur mise en culture, tous les fragments d'entre-nœuds commencent à émettre des cals blanchâtres plus ou moins volumineux. Ils apparaissent tout d'abord aux deux extrémités des fragments avant d'émerger des lenticelles et recouvrir la totalité des explants. Détachés des entrenœuds qui les ont engendrés, et régulièrement repiqués sur un milieu frais, ces cals continuent à proliférer, prennent un aspect granuleux et deviennent chlorophylliens. Maintenus 5 à 7 semaines sans repiquage, un petit nombre d'entre eux (3\%) produisent à leur surface de petites boursouflures se détachant avec facilité. La plupart d'entre elles évoluent rapidement en embryons somatiques.

Chez le chêne vert, les premiers cals apparaissent au bout de 4 jours. Après 7 mois de culture sans aucun repiquage, à la surface des cals s'individualisent de petites protubérances sphériques. Ces "nodules primaires" sont repiqués un à un. Exposés à la lumière, ils prolifèrent en cals chlorophylliens sans aucune organogenèse. Quelques-uns (3\%) de ceux cultivés à l'obscurité produisent de nouvelles protubérances ou "nodules secondaires" qui repiqués aussi bien à la lumière qu'à l'obscurité produisent des embryons somatiques.

Chez le chêne liège et le chêne pubescent, les embryons zygotiques excisés à des stades très précoces de leur développement se nécrosent totalement. Prélevés au contraire à un stade proche de la maturité, ils poursuivent leur développement "normal" en une jeune plante. Aux stades intermédiaires, ils produisent des embryons somatiques; le phénomène est généralisé et affecte toute leur surface pour les plus jeunes ou bien se localise à l'extrémité radiculaire chez ceux qui étaient un peu plus avancés dans leur développement. Dans tous les cas, chaque embryon somatique se forme à partir d'une cellule superficielle de l'embryon zygotique, sans qu'il y ait, à quelque moment que ce soit, mise en place d'un cal.

Chez les trois espèces que nous avons testées tous les embryons somatiques obtenus ont produit en quantité importante des embryons secondaires. Ce phénomène comparable à ce qui a été décrit par Maheswaran et Williams (1985) chez Trifolium repens ou par Michaux-Ferrière et al. (1987) chez Coffea arabica met donc en place une "embryogenèse somatique adventive" qui, dans le cas de nos chênes au moins, se poursuit apparemment de façon indéfinie.

\section{Discussion}

On retrouve chez le chêne les deux modalités d'embryogenèse somatique décrites par les auteurs. Directe, à partir des embryons zygotiques immatures de $Q$. suber ou $Q$. pubescens, sans aucun doute parce que ces embryons sont entièrement constitués de cellules méristématiques. Indirecte, à partir des fragments d'entrenœuds de $Q$. suber ou de pétioles de $Q$. ilex; dans ces deux cas, en effet, le phénomène nécessite la mise en place préalable d'un cal. Bien que ces résultats concernent deux espèces différentes, il n'est pas sans intérêt de noter que : 1) dans nos expériences, l'obtention des 
embryons a été plus rapide (7 semaines) à partir de cals fournis par des fragments prélevés sur des individus jeunes ( 8 mois) qu'à partir de ceux mis en place par des pétioles récoltés sur des arbres de 50 ans; 2) dans les deux cas d'embryogenèse indirecte, le repiquage régulier des cals exerce un rôle inhibiteur sur la mise en place de l'embryogenèse somatique. On peut penser que la fragmentation pratiquée au moment de la transplantation relance la prolifération et empêche ainsi le cal de s'engager dans la voie de la morphogénèse.

Dans nos expériences, l'obtention d'embryons somatiques par voie indirecte est quelque peu aléatoire ( $3 \%$ des cas). Ceci sans aucun doute parce que nous n'utilisons pas de 2,4-D, contrairement à ce qui se fait souvent. Cette situation est en quelque sorte compensée par l'installation d'une embryogenèse somatique adventive qui fait que l'on se trouve en présence d'une embryogenèse continue et apparemment illimitée. II faut certainement rapprocher ce phénomène des résultats obtenus chez Coffea arabica (Yasuda et al., 1985) et chez Aesculus hippocastanum (Dameri et al., 1986). Toutefois, cette production d'embryons en cascade s'oppose, chez nos trois espèces, au développement des em- bryons en plantes feuillées et représente donc un obstacle à leur utilisation dans un processus de propagation.

\section{Références}

Dameri R.M., Caffaro L., Gastaldo P. \& Profumo P. (1986) Callus formation and embryogenesis with leaf explants of Aesculus hippocastanum L. J. Plant Physiol. 126, 93-96

El Maâtaoui M. \& Espagnac H. (1987) Néoformation de structures de type embryons somatiques sur des cultures de tissus de chêne liège (Quercus suber L.). C.R. Acad. Sci. Paris Ser. III $304,83-88$

Féraud-Keller C. \& Espagnac H. (1989) Conditions d'apparition d'une embryogenèse somatique sur des cals issus de culture de tissus foliaires de chêne vert (Quercus ilex L.). Can. J. Bot. $67,1066-1070$

Maheswaran G. \& Williams E.G. (1985) Origin and development of somatic embryoids formed directly on immature embryos of Trifolium repens in vitro. Ann. Bot. 56, 619-630

Michaux-Ferrière N., Dublin P. \& Schwendiman J. (1987) Elude histologique de l'embryogenèse somatique a partir d'explants foliaires de Coffea arabica L. Café Cacao Thé 31, 103-114

Yasuda T., Fujii Y. \& Yamaguchi T. (1985) Embryogenic callus induction from Coffea arabica leaf explants by benzyladenine. Plant Cell Physiol. 26, 595-597 